\title{
New aspects of impaired mitochondrial function in heart failure
}

\author{
Mariana G. Rosca $\cdot$ Charles L. Hoppel \\ Published online: 4 April 2009 \\ (C) The Author(s) 2009. This article is published with open access at Springerlink.com
}

\begin{abstract}
This minireview focuses on the impairment of function in cardiac mitochondria in heart failure (HF). It is generally accepted that chronic energy starvation leads to cardiac mechanical dysfunction in HF. Mitochondria are the primary ATP generator for the heart. Current evidence suggests that the assembly of the electron transport chain (ETC) into respirasomes provides structural support for mitochondrial oxidative phosphorylation by facilitating electron channeling and perhaps by preventing electron leak and superoxide production. Defects have been purported to occur in the individual ETC complexes or components of the phosphorylation apparatus in HF, but these defects have not been linked to impaired mitochondrial function. Moreover, studies that reported decreased mitochondrial oxidative phosphorylation in HF did not identify the site of the defect. We propose a sequential mechanistic pathway in which the decrease in functional respirasomes in HF is the primary event causing decreased oxidative phosphorylation and increased reactive oxygen species production, leading to a progressive decrease in cardiac performance.
\end{abstract}

Keywords Heart failure · Mitochondria · Respirasomes · Oxidative phosphorylation

\footnotetext{
M. G. Rosca · C. L. Hoppel

Center for Mitochondrial Diseases and Departments of Medicine and Pharmacology, Case Western Reserve University,

Cleveland, OH, USA

C. L. Hoppel $(\triangle)$

Department of Pharmacology, Case Western Reserve University,

School of Medicine,

10900 Euclid Avenue,

Cleveland, OH 44106-4981, USA

e-mail: charles.hoppel@case.edu
}

\section{Introduction}

Heart failure (HF) is a multifactorial syndrome characterized by mechanical dysfunction of the myocardium and by failure to meet the perfusion requirement of the body. Both cardiac contraction and relaxation have a continuous energy demand. Decreased levels of high-energy phosphates are found in cardiac muscle in advanced stages of HF (Beer et al. 2002; Neubauer et al. 1997; Sanbe et al. 1995). HF changes all components of cardiac energetics: substrate utilization, ATP production, and ATP transfer to the cardiac contractile apparatus (Neubauer 2007; Stanley et al. 2005). The metabolic fuel shift in substrate preference in the failing heart from fatty acid to glucose (Davila-Roman et al. 2002; Massie et al. 1995) suggests that the main sources for the reducing equivalents, $\mathrm{NADH}$ and $\mathrm{FADH}_{2}$, become glycolysis and glucose oxidation. The progressive decrease in the amount of ATP in the heart (Shen et al. 1999) linked with reduction in mitochondrial respiration (Rosca et al. 2008; Sharov et al. 1998; Sharov et al. 2000) and mitochondrial structural abnormalities (Ide et al. 2001; Sabbah et al. 1992) indicates that the mitochondrial ATP production also decreases in the failing heart. The transfer of mitochondrial ATP via the creatine-kinase energy shuttle to myofibrillar actomyosin ATPase also is decreased (Neubauer et al. 1999). Consequently, the failing heart is an energy-starved engine (Neubauer 2007) so that improvement of myocardial energetics becomes a promising approach to the treatment of HF. This minireview addresses the available data that support the contribution of mitochondrial dysfunction to the progression of HF.

\section{Respirasomes as structural support for oxidative phosphorylation in heart mitochondria}

The conventional model for electron transport between the four mitochondrial independent complexes connected by 
electron carriers that randomly diffuse within the inner membrane ("fluid model") (Hackenbrock et al. 1986) has been modified. In the alternate "solid model" initially proposed by Chance and Williams more than 50 years ago (Chance and Williams. 1955) and expanded and amplified by Schagger's group (Schagger and Pfeiffer 2000), the ETC complexes are assembled into high-molecular weight supercomplexes. The supercomplex consisting of complex I, dimeric complex III, and one copy of complex IV $\left(\mathrm{I}_{1} \mathrm{III}_{2} \mathrm{IV}_{1}\right.$ ) found in rodent (Wittig et al. 2006), dog (Rosca et al. 2008), and bovine (Schagger and Pfeiffer. 2000) heart mitochondria, as well as in plants (Krause et al. 2004) and fungi (Boumans et al. 1998), also contains coenzyme Q and cytochrome c, and functions as a cohesive respiratory unit (respirasome) because it transports electrons from NADH to reduce oxygen (Acin-Perez et al. 2008). Functional complex II also is associated with the respirasome $\mathrm{I}_{1} \mathrm{II}_{1} \mathrm{III}_{2} \mathrm{IV}_{1}$ which oxidizes both $\mathrm{NADH}$ and $\mathrm{FADH}_{2}$ and reduces oxygen to form water (Acin-Perez et al. 2008).

As expressed by the chemiosmotic theory, the chemical energy of the electron transfer down the redox potential gradient from NADH and $\mathrm{FADH}_{2}$ through the ETC components to oxygen is harnessed as an inner membrane electrochemical gradient (Mitchell 1976)), which drives the synthesis of ATP by ATP synthase. How does this mitochondrial function benefit from the structural organization of the ETC into respirasomes?

On the basis of structural information obtained from the three-dimensional map of the bovine heart $\mathrm{I}_{1} \mathrm{III}_{2} \mathrm{IV}_{1}$ supercomplex (Schafer et al. 2007), the individual complexes physically interact in this assembly. Dimeric complex III is associated with the inner membrane arm of complex I, whereas monomeric complex IV is associated with both complex III and the membrane arm of complex I. The electron carrier-binding sites of one complex are both in proximity to and face the electron carrier-binding sites of the subsequent complex. In this multienzyme assembly, NADH dehydrogenase localized in the matrix arm of the Lshaped complex I oxidizes NADH, with the two electrons and two protons accepted by the flavin mononucleotide. Electrons are further transferred individually via a series of iron-sulfur centers to reduce coenzyme $\mathrm{Q}$ at a binding site near the connection between the matrix and inner membrane arms of complex I. Reduced coenzyme Q (ubiquinol) then binds to an adjacent site in complex III and transfers electrons to the Rieske iron-sulfur protein and cytochrome b. The transfer of electrons through complex III is best explained by the $\mathrm{Q}$ cycle, in which four protons are translocated across the inner membrane for every two electrons transferred from ubiquinol to cytochrome $c_{1}$ to reduce cytochrome c. As the last step, cytochrome c oxidase (COX) reduces oxygen to water upon oxidation of cytochrome $\mathrm{c}$ and translocation of protons through the inner membrane. The cytochrome c binding site of COX represented by the globular domain of COX subunits II faces the cytochrome $\mathrm{c}$ binding site in complex III (cytochrome $\mathrm{c}_{1}$ ). According to this model, the electron carriers have short diffusion distances between complexes, supporting the concept of channeled electron transfer decreasing electron leakage and superoxide production (Vonck and Schafer. 2009).

Conversion of the electrochemical gradient created by the ETC to high energy ATP is carried out by the $F_{1} F_{0}$ ATP synthase (complex V). ATP synthase also joins components of the phosphorylation apparatus (adenine nucleotide translocase and phosphate transporter) to form supramolecular structures called synthasomes (Chen et al. 2004). Associations of complex $\mathrm{V}$ with supercomplexes co-exist with assemblies with individual ETC complexes (Acin-Perez et al. 2008), but the functional significance of these associations has not been established.

More recently, a "plasticity model" for the organization of respiratory chain was proposed that accommodates the coexistence of individual complexes with supercomplexes, whose composition varies according to the bioenergetic demand (Acin-Perez et al. 2008). These observations suggest that the organization of the mitochondrial inner membrane creates a platform for the coordination of electron transport with ATP synthesis.

\section{The approach to heart mitochondria in $\mathrm{HF}$}

Activity of individual components of the respiratory chain

A large variety of mitochondrial defects has been described in the ETC complexes and components of the phosphorylation apparatus in both humans and experimental models of HF. Sparagna et al. reported a decrease in complex IV activity in spontaneously hypertensive rats, an experimental model of pressure overload HF (Sparagna et al. 2007). Using the pacing-induced canine model of HF, Ide et al. reported a decrease in complex I activity (Ide et al. 1999), whereas Marin-Garcia et al. reported a severe decrease in the activity of complex III (Marin-Garcia et al. 2001a, b; Moe et al. 2004). Decreased activities of complexes III and IV have been reported in human patients with congestive HF (Buchwald et al. 1990; Jarreta et al. 2000). Alterations in the components of the phosphorylation apparatus characterized by decreased amount and activity of ATP synthase were reported to occur in pigs with ischemic HF induced by left circumflex coronary artery ligation (Liu et al. 2001) as well as in dogs with pacing-induced HF (Marin-Garcia et al. 2001a, b; Moe et al. 2004), dogs with naturally occurring dilated cardiomyopathy (McCutcheon et al. 1992), and human patients with dilated cardiomyopathy (Unverferth et al. 1988). Dorner et al. reported a switch in 
the amount of adenine nucleotide translocase (ANT) protein characterized by an increase in the ANT1 isoform and a decrease in ANT2, associated with a decrease in the nucleotide transport activity in patients with idiopathic dilated cardiomyopathy (Dorner et al. 2006) as well as with enteroviral myocarditis (Dorner et al. 2000).

However, a direct causal relationship between the decreased activity of ETC complexes and HF has proven elusive (Jarreta et al. 2000). In addition, the link between the reported specific defects and the decrease in mitochondrial function has not been studied. There is an apparent excess in the activity of the ETC complexes and phosphorylation apparatus components relative to the oxidative phosphorylation needs (Faustin et al. 2004; Rossignol et al. 1999). The sites of control of respiration in normal heart mitochondria are located at complex I in the ETC (Groen et al. 1982) and at the adenine nucleotide translocase and complex V in the phosphorylation apparatus (Gellerich et al. 1983). Therefore, flux control analysis is necessary in heart mitochondria from heart failure to link the ETC defects with decreased oxidative phosphorylation in HF.

Integrative mitochondrial function

The integrated function of the ETC coupled to ATP synthesis, membrane transport, dehydrogenase activities, and the structural integrity of the mitochondria is assessed by analysis of oxidative phosphorylation performed in either saponin-permeabilized fibers or in freshly-isolated mitochondria. Decreased state 3 respiratory rates (ADPdependent) were reported when oxygen consumption was measured in the presence of glutamate + malate in saponinpermeabilized cardiac fibers isolated from rat (Garnier et al. 2003; Sanbe et al. 1994), dog (Sharov et al. 1998) and human (Sharov et al. 2000) hearts with dilated cardiomyopathy as well as pressure overload and ischemic HF (Sanbe et al. 1993; Sharov et al. 2000). However, oxygen consumption by the swine myocardium with pressure overload congestive HF was unchanged (Gong et al. 2003). The decrease of the respiratory rates measured in cardiac fibers may be attributed to either a decrease in mitochondrial density or a decrease in mitochondrial function. The decrease in mitochondrial density was ruled out as a cause for the decrease in respiratory rates in dogs with intracoronary-microembolization HF (Sharov et al. 1998). In contrast, Garnier et al reported a discrepancy between the decreased citrate synthase activity and unchanged mitochondrial DNA copy number as indicators of cardiac mitochondrial content (Garnier et al. 2003).

Studies on oxidative phosphorylation of freshly-isolated heart mitochondria in HF show that the two topologically and biochemically distinct mitochondrial populations, subsarcolemmal (SSM) and interfibrillar (IFM) mitochondria
(Palmer et al. 1977; Palmer et al. 1985) are selectively affected by HF of differing etiology. Initial studies on oxidative phosphorylation in freshly-isolated heart mitochondria were performed on SSM. Lindenmayer et al. reported dramatic decreases in state 3 respiratory rates during glutamate and succinate oxidation in heart SSM isolated from guinea pigs with congestive HF induced by stenosis of the ascending aorta (Lindenmayer et al. 1968). A similar model of HF in rabbits was associated with only a $22 \%$ decrease in state 3 respiratory rates of heart mitochondria oxidizing either glutamate + malate or succinate (Sordahl et al. 1973). However, the use of the protease, nagarse, complemented by polytron homogenization means that these authors isolated both heart SSM and IFM as a single admixed fraction. These data showing a milder decrease in state 3 respiratory rates compared with the previous study are based on a mixture of the two types of heart mitochondria populations differently affected by HF, a circumstance that would partially mask the severity of the defect. Of interest, density gradient analysis of the isolated mitochondria revealed a greater heterogeneity in mitochondrial size in HF compared to the control, with two morphologically distinct mitochondrial populations emerging in the congestive HF stage. Biochemical studies of these two individual peaks have not been done. The control of oxygen consumption by phosphorylation ("coupling") is lost in pressure overload congestive HF as indicated by the decreased respiratory control ratio (state 3 rate/state 4 rate (ADP-independent)) (Lindenmayer et al. 1968; Sordahl et al. 1973) with little or no change in the efficiency of oxidative phosphorylation, as shown by normal ADP/O ratio (Lindenmayer et al. 1968; Sordahl et al. 1973). The specific site of the mitochondrial defect was not identified in these studies. The analysis of mitochondrial oxidative phosphorylation was performed with a limited number of substrates, and was not supplemented by the measurement of the activities of the individual ETC complexes.

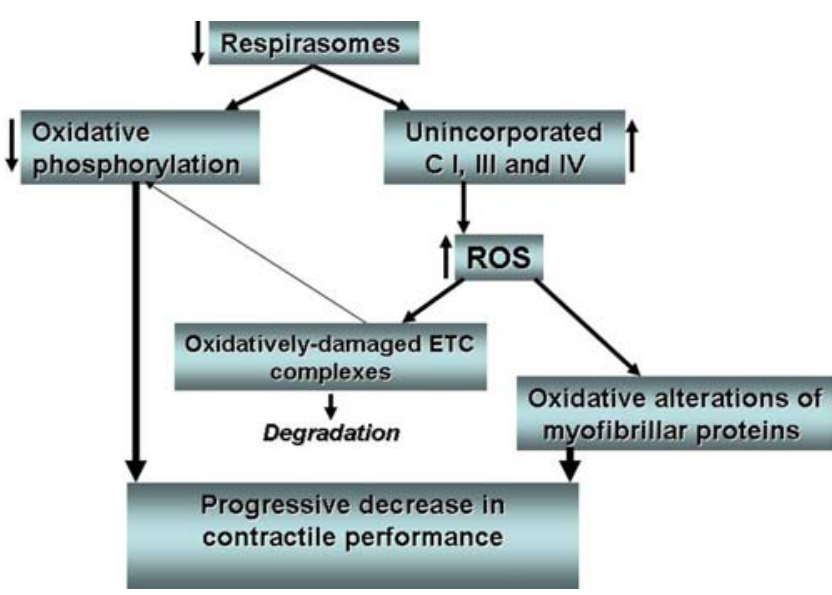

Fig. 1 The sequence of mitochondrial changes in heart failure 
Dystrophic cardiomyopathy in hamsters also was reported to be associated with decreased oxidation of glutamate and succinate by heart mitochondria (Lindenmayer et al. 1970), whereas abnormalities in oxidative phosphorylation were not detected in other studies of the same pathological condition (Wrogemann et al. 1972). These disparate findings were reconciled when the two metabolically distinct populations of mitochondria in the hamster heart were separately described microscopically and biochemically (Hoppel et al. 1982). A decrease in respiratory state 3 rates with complex I or II substrates was present only in IFM. The activity of enzymes involved in the generation of reducing equivalents to the ETC, as well as the activities of ETC complexes and the amount of cytochromes, was normal. The decrease in state 3 respiratory rates was reversed by addition of an uncoupler, indicating that the defect lay in the phosphorylation apparatus. The control of oxygen consumption by phosphorylation ("coupling") as well as the efficiency of oxidative phosphorylation was preserved. The defect in IFM was progressive and paralleled the degree of peripheral congestion. In this study, the assay of oxidative phosphorylation on freshly-isolated intact mitochondria was the only test able to localize the defect.

\section{Respirasomes and oxidative phosphorylation in HF}

In a follow-up to the work of Sharov et al. on oxidative phosphorylation in saponin-permeabilized cardiac fibers (Sharov et al. 1998), we performed a comprehensive study of respiratory properties of the two populations of heart mitochondria (Rosca et al. 2008) in the canine model of intracoronary microembolization-induced HF of moderate severity. Both populations of heart mitochondria were equally affected in this model of HF. We found a dramatic decrease in the ADP-stimulated respiration that was not relieved by an uncoupler, indicating that the defect in oxidative phosphorylation is localized in the ETC. The decrease in ADP-stimulated respiration with substrates that donate electrons to complexes I, II, III, and IV suggests a defect localized in cytochrome c oxidase (COX). However, neither COX activity nor the amount of the substrate cytochrome $\mathrm{c}$ were altered in HF. The amount of $\mathrm{I}_{1} \mathrm{III}_{2} \mathrm{IV}_{1}$ respirasomes was decreased in both populations of heart mitochondria. We concluded that the decrease in oxidative phosphorylation of heart mitochondria can be explained by the decrease in the amount of functional respirasomes. Also, the decrease in succinate oxidation in HF with unchanged complex II activity suggests that the electron flow from complex II supplies the complex III incorporated into $\mathrm{I}_{1} \mathrm{III}_{2} \mathrm{IV}_{1}$ respirasomes rather than the un-incorporated complex III.

\section{Mitochondria in HF: a matter of assembly}

Based on our observations, we propose a sequential mechanistic pathway by which mitochondrial alterations contribute to the progression of HF (Fig. 1). The decrease in functional respirasomes in $\mathrm{HF}$ is the primary event causing the decrease in oxidative phosphorylation coupled with an increase in electron leakage and superoxide generation in complexes I and III that are independent of respirasomes. Complex I-generated superoxide is dismutated by matrix manganese superoxide dismutase (MnSOD) to diffusible hydrogen peroxide, which damages adjacent mitochondrial inner membrane proteins including subunits of the ETC complexes. These oxidative alterations cause decreased activity of ETC complexes reported by others in severe HF, potentially decrease oxidative phosphorylation, and finally lead to the degradation of the oxidized complexes. Complex III-generated superoxide that exceeds the dismutation capacity of the intermembrane space $\mathrm{Cu}-\mathrm{Zn}$ SOD, exits the mitochondria through the outer membrane voltage-dependent anion channels and is dismutated to hydrogen peroxide within the cytosol. The oxidative modifications of cytosolic myofibrillar proteins lead to the progressive decrease in their contractile performance and signal the transition to irreversible heart damage (Fig. 1). This scenario is supported by the observation that the increased oxidative stress in mice with MnSOD deficiency targeted to the heart (Nojiri et al. 2006) causes decreased activity of mitochondrial ETC complexes and progressive dilated cardiomyopathy.

Acknowledgments Funding for this work was provided by the National Heart, Lung and Blood Institute, Program Project Grant PO1 (HL074237). We thank Drs. Tatyana Klimova and Bernard Tandler for editorial assistance.

Open Access This article is distributed under the terms of the Creative Commons Attribution Noncommercial License which permits any noncommercial use, distribution, and reproduction in any medium, provided the original author(s) and source are credited.

\section{References}

Acin-Perez R, Fernandez-Silva P, Peleato ML, Perez-Martos A, Enriquez JA (2008) Respiratory active mitochondrial supercomplexes. Molecular cell 32:529-539

Beer M, Seyfarth T, Sandstede J, Landschutz W, Lipke C, Kostler H, von Kienlin M, Harre K, Hahn D, Neubauer S (2002) Absolute concentrations of high-energy phosphate metabolites in normal, hypertrophied, and failing human myocardium measured noninvasively with (31) P-SLOOP magnetic resonance spectroscopy. J Am Coll Cardiol 40:1267-1274

Boumans H, Grivell LA, Berden JA (1998) The respiratory chain in yeast behaves as a single functional unit. J Biol Chem 273:4872-4877

Buchwald A, Till H, Unterberg C, Oberschmidt R, Figulla HR, Wiegand V (1990) Alterations of the mitochondrial respiratory 
chain in human dilated cardiomyopathy. Eur Heart J 11:509_ 516

Chance B, Williams GR (1955) A method for the localization of sites for oxidative phosphorylation. Nature 176:250-254

Chen C, Ko Y, Delannoy M, Ludtke SJ, Chiu W, Pedersen PL (2004) Mitochondrial ATP synthasome: three-dimensional structure by electron microscopy of the ATP synthase in complex formation with carriers for Pi and ADP/ATP. J Biol Chem 279:3176131768

Davila-Roman VG, Vedala G, Herrero P, de las Fuentes L, Rogers JG, Kelly DP, Gropler RJ (2002) Altered myocardial fatty acid and glucose metabolism in idiopathic dilated cardiomyopathy. J Am Coll Cardiol 40:271-277

Dorner A, Giessen S, Gaub R, Grosse Siestrup H, Schwimmbeck PL, Hetzer R, Poller W, Schultheiss HP (2006) An isoform shift in the cardiac adenine nucleotide translocase expression alters the kinetic properties of the carrier in dilated cardiomyopathy. Eur J Heart Fail 8:81-89

Dorner A, Pauschinger M, Schwimmbeck PL, Kuhl U, Schultheiss HP (2000) The shift in the myocardial adenine nucleotide translocator isoform expression pattern is associated with an enteroviral infection in the absence of an active T-cell dependent immune response in human inflammatory heart disease. J Am Coll Cardiol 35:1778-1784

Faustin B, Rossignol R, Rocher C, Benard G, Malgat M, Letellier T (2004) Mobilization of adenine nucleotide translocators as molecular bases of the biochemical threshold effect observed in mitochondrial diseases. J Biol Chem 279:20411-20421

Garnier A, Fortin D, Delomenie C, Momken I, Veksler V, VenturaClapier R (2003) Depressed mitochondrial transcription factors and oxidative capacity in rat failing cardiac and skeletal muscles. J Physiol 551:491-501

Gellerich FN, Bohnensack R, Kunz W (1983) Control of mitochondrial respiration. The contribution of the adenine nucleotide translocator depends on the ATP-and ADP-consuming enzymes. Biochimica et biophysica acta 722:381-391

Gong G, Liu J, Liang P, Guo T, Hu Q, Ochiai K, Hou M, Ye Y, Wu X, Mansoor A, From AH, Ugurbil K, Bache RJ, Zhang J (2003) Oxidative capacity in failing hearts. Am J Physiol 285:H541-548

Groen AK, Wanders RJ, Westerhoff HV, van der Meer R, Tager JM (1982) Quantification of the contribution of various steps to the control of mitochondrial respiration. J Biol Chem 257:2754-2757

Hackenbrock CR, Chazotte B, Gupte SS (1986) The random collision model and a critical assessment of diffusion and collision in mitochondrial electron transport. J Bioenerg Biomembr 18:331-368

Hoppel CL, Tandler B, Parland W, Turkaly JS, Albers LD (1982) Hamster cardiomyopathy. A defect in oxidative phosphorylation in the cardiac interfibrillar mitochondria. J Biol Chem 257:1540 1548

Ide T, Tsutsui H, Hayashidani S, Kang D, Suematsu N, Nakamura K, Utsumi H, Hamasaki N, Takeshita A (2001) Mitochondrial DNA damage and dysfunction associated with oxidative stress in failing hearts after myocardial infarction. Circ Res 88:529-535

Ide T, Tsutsui H, Kinugawa S, Utsumi H, Kang D, Hattori N, Uchida K, Arimura K, Egashira K, Takeshita A (1999) Mitochondrial electron transport complex I is a potential source of oxygen free radicals in the failing myocardium. Circ Res 85:357-363

Jarreta D, Orus J, Barrientos A, Miro O, Roig E, Heras M, Moraes CT, Cardellach F, Casademont J (2000) Mitochondrial function in heart muscle from patients with idiopathic dilated cardiomyopathy. Cardiovasc Res 45:860-865

Krause F, Reifschneider NH, Vocke D, Seelert H, Rexroth S, Dencher NA (2004) "Respirasome"-like supercomplexes in green leaf mitochondria of spinach. J Biol Chem 279:48369-48375

Lindenmayer GE, Harigaya S, Bajusz E, Schwartz A (1970) Oxidative phosphorylation and calcium transport of mitochondria isolated from cardiomyopathic hamster hearts. J Mol Cell Cardiol 1:249259

Lindenmayer GE, Sordahl LA, Schwartz A (1968) Reevaluation of oxidative phosphorylation in cardiac mitochondria from normal animals and animals in heart failure. Circ Res 23:439-450

Liu J, Wang C, Murakami Y, Gong G, Ishibashi Y, Prody C, Ochiai K, Bache RJ, Godinot C, Zhang J (2001) Mitochondrial ATPase and high-energy phosphates in failing hearts. Am J Physiol 281: H1319-1326

Marin-Garcia J, Goldenthal MJ, Moe GW (2001a) Abnormal cardiac and skeletal muscle mitochondrial function in pacing-induced cardiac failure. Cardiovasc Res 52:103-110

Marin-Garcia J, Goldenthal MJ, Moe GW (2001b) Mitochondrial pathology in cardiac failure. Cardiovasc Res 49:17-26

Massie BM, Schaefer S, Garcia J, McKirnan MD, Schwartz GG, Wisneski JA, Weiner MW, White FC (1995) Myocardial highenergy phosphate and substrate metabolism in swine with moderate left ventricular hypertrophy. Circulation 91:18141823

McCutcheon LJ, Cory CR, Nowack L, Shen H, Mirsalami M, Lahucky R, Kovac L, O'Grady M, Horne R, O’Brien PJ (1992) Respiratory chain defect of myocardial mitochondria in idiopathic dilated cardiomyopathy of Doberman pinscher dogs. Can J Physiol Pharmacol 70:1529-1533

Mitchell P (1976) Possible molecular mechanisms of the protonmotive function of cytochrome systems. J Theor Biol 62:327-367

Moe GW, Marin-Garcia J, Konig A, Goldenthal M, Lu X, Feng Q (2004) In vivo TNF-alpha inhibition ameliorates cardiac mitochondrial dysfunction, oxidative stress, and apoptosis in experimental heart failure. Am J Physiol 287:H1813-1820

Neubauer S (2007) The failing heart-an engine out of fuel. N Engl J Med 356:1140-1151

Neubauer S, Horn M, Cramer M, Harre K, Newell JB, Peters W, Pabst T, Ertl G, Hahn D, Ingwall JS, Kochsiek K (1997) Myocardial phosphocreatine-to-ATP ratio is a predictor of mortality in patients with dilated cardiomyopathy. Circulation 96:2190-2196

Neubauer S, Remkes H, Spindler M, Horn M, Wiesmann F, Prestle J, Walzel B, Ertl G, Hasenfuss G, Wallimann T (1999) Downregulation of the $\mathrm{Na}(+)$-creatine cotransporter in failing human myocardium and in experimental heart failure. Circulation 100:1847-1850

Nojiri H, Shimizu T, Funakoshi M, Yamaguchi O, Zhou H, Kawakami S, Ohta Y, Sami M, Tachibana T, Ishikawa H, Kurosawa H, Kahn RC, Otsu K, Shirasawa T (2006) Oxidative stress causes heart failure with impaired mitochondrial respiration. J Biol Chem 281:33789-33801

Palmer JW, Tandler B, Hoppel CL (1977) Biochemical properties of subsarcolemmal and interfibrillar mitochondria isolated from rat cardiac muscle. J Biol Chem 252:8731-8739

Palmer JW, Tandler B, Hoppel CL (1985) Biochemical differences between subsarcolemmal and interfibrillar mitochondria from rat cardiac muscle: effects of procedural manipulations. Arch Biochem Biophys 236:691-702

Rosca MG, Vazquez EJ, Kerner J, Parland W, Chandler MP, Stanley W, Sabbah HN, Hoppel CL (2008) Cardiac mitochondria in heart failure: decrease in respirasomes and oxidative phosphorylation. Cardiovasc Res

Rossignol R, Malgat M, Mazat JP, Letellier T (1999) Threshold effect and tissue specificity. Implication for mitochondrial cytopathies. J Biol Chem 274:33426-33432

Sabbah HN, Sharov V, Riddle JM, Kono T, Lesch M, Goldstein S (1992) Mitochondrial abnormalities in myocardium of dogs with chronic heart failure. J Mol Cell Cardiol 24:1333-1347

Sanbe A, Tanonaka K, Hanaoka Y, Katoh T, Takeo S (1993) Regional energy metabolism of failing hearts following myocardial infarction. J Mol Cell Cardiol 25:995-1013 
Sanbe A, Tanonaka K, Kobayasi R, Takeo S (1995) Effects of longterm therapy with ACE inhibitors, captopril, enalapril and trandolapril, on myocardial energy metabolism in rats with heart failure following myocardial infarction. J Mol Cell Cardiol 27:2209-2222

Sanbe A, Tanonaka K, Niwano Y, Takeo S (1994) Improvement of cardiac function and myocardial energy metabolism of rats with chronic heart failure by long-term coenzyme Q10 treatment. J Pharmacol Exp Ther 269:51-56

Schafer E, Dencher NA, Vonck J, Parcej DN (2007) Threedimensional structure of the respiratory chain supercomplex I1III2IV1 from bovine heart mitochondria. Biochemistry 46:12579-12585

Schagger H, Pfeiffer K (2000) Supercomplexes in the respiratory chains of yeast and mammalian mitochondria. EMBO J 19:1777-1783

Sharov VG, Goussev A, Lesch M, Goldstein S, Sabbah HN (1998) Abnormal mitochondrial function in myocardium of dogs with chronic heart failure. J Mol Cell Cardiol 30:1757-1762

Sharov VG, Todor AV, Silverman N, Goldstein S, Sabbah HN (2000) Abnormal mitochondrial respiration in failed human myocardium. J Mol Cell Cardiol 32:2361-2367

Shen W, Asai K, Uechi M, Mathier MA, Shannon RP, Vatner SF, Ingwall JS (1999) Progressive loss of myocardial ATP due to a loss of total purines during the development of heart failure in dogs: a compensatory role for the parallel loss of creatine. Circulation 100:2113-2118

Sordahl LA, McCollum WB, Wood WG, Schwartz A (1973) Mitochondria and sarcoplasmic reticulum function in cardiac hypertrophy and failure. Am J Physiol 224:497-502

Sparagna GC, Chicco AJ, Murphy RC, Bristow MR, Johnson CA, Rees ML, Maxey ML, McCune SA, Moore RL (2007) Loss of cardiac tetralinoleoyl cardiolipin in human and experimental heart failure. J Lipid Res 48:1559-1570

Stanley WC, Recchia FA, Lopaschuk GD (2005) Myocardial substrate metabolism in the normal and failing heart. Physiological reviews $85: 1093-1129$

Unverferth DV, Lee SW, Wallick ET (1988) Human myocardial adenosine triphosphatase activities in health and heart failure. Am Heart J 115:139-146

Vonck J, Schafer E (2009) Supramolecular organization of protein complexes in the mitochondrial inner membrane. Biochimica et biophysica acta 1793:117-124

Wittig I, Carrozzo R, Santorelli FM, Schagger H (2006) Supercomplexes and subcomplexes of mitochondrial oxidative phosphorylation. Biochimica et biophysica acta 1757:1066-1072

Wrogemann K, Blanchaer MC, Jacobson BE (1972) Oxidative phosphorylation in cardiomyopathic hamsters. Am J Physiol 222:1453-1457 